\title{
Nursing Leadership Strategies for Implementing Nursing Full Scope of Practice into Acute Care Team-Led Healthcare Settings
}

\author{
Alexandra Jean Hamilton ${ }^{1}$, Elke Huber ${ }^{2}$, Ayah Shakshuki ${ }^{3}$ and Andrea LO Hebb*2,4 \\ ${ }^{1}$ Saint Francis Xavier School of Nursing, St Francis Xavier University, USA \\ ${ }^{2}$ Dalhousie University School of Nursing, University Avenue, USA \\ ${ }^{3}$ Dalhousie University College of pharmacy, College of pharmacy, USA \\ ${ }^{4}$ Dalhousie University, Division of Neurosurgery, USA
}

Received: March 26, 2018; Published: April 12, 2018

*Corresponding author: Andrea LO Hebb, Saint Francis Xavier School of Nursing 2340 Notre Dame Avenue St Francis Xavier University, Antigonish, Dalhousie University, Division of Neurosurgery, Nova Scotia Health Authority, Halifax, Canada, USA, Email: alohebb@dal.ca

\section{Introduction}

The nursing new model of care was introduced in Nova Scotia as an innovative cost-efficient measure to integrate registered nurses (RN) and licensed practical nurses (LPN) in a unified strategy as a team-led holistic initiative to produce best patient outcomes. Such an approach to reorganizing nursing practice necessitates RNs to work to their full scope of practice. Working to full scope has been interpreted by tasks nurses perform and not what professional regulatory bodies dictate. There is increased RN-LPN tension when LPNs are working to full scope and tasks overlap, a pay differential still occurs. Working to full scope has implications for nursing leadership to sustain healthy work environments. The incentive driving creation of innovative nursing practice models is to contain costs as well as promote retention and recruitment of nurses in acute care [1]. Research has only recently addressed the question of the impact of the models' effects on patient outcomes [2].

\section{The Issue and Implications for Nursing Practice and} Leadership

The issue is two parts. First nurses do not work to full scope of practice and feel their skills are not recognized or valued CNAC [3]. Second, nurses who are practicing full scope have expressed concern and resentment. For instance, in a hospital environment, certain units may primarily employ RNs; however others have teams consisting primarily of LPNs which may result in hostility and resentment from RNs [4]. Concerns encompass the possibility of job loss, territoriality, misperceptions about the role of the LPN, a resistance to change and role confusion. The client, scope of practice and practice environment may be factors determining patient assignment in acute care settings CRNNS [5] and CLPNNS [6]. More of the traditional nursing tasks are being handed to
LPNs while RNs are acquiring increased high acuity patients with complex healthcare needs [7]. Scope of practice for LPNs in Nova Scotia includes assessment and evaluating patients, collaborating in developing and implementing a nursing care plan CLPNNS [6]. LPNs may practice independently given the patient(s) need(s) are not complex and are predictable CRNNS [8].

However, the responsibilities and extent to which duties are performed varies based on employer policies, the practice environment, and of course the complexity of the patient. Complex patients whom are considered to have unpredictable health status are mainly handled by RNs or LPNs under the direction of an RN or another healthcare professional CRNNS [8]. Implications for nursing practice include support of health organizations in recognizing the full scope of nursing practice to exploit cost-efficient use of human resources [9]. Leadership roles involve establishing a positive work environment, scope clarity, team-building and practice opportunities [10]. Emotional intelligence as a basis for nursing leadership has been shown to have a positive outcome in the work environment as it supports professional nursing practice and teamwork between health care providers [11]. Effective leadership comes with experience and without it deficiencies in care have been presented [11]. Leadership competencies/skills needed to respond effectively to changing models of care require effective decisionmaking to lead and sustain change (Appendix 1).

\section{Historical Background of the Issue, Alternative Explana- tions and Associated Assumptions}

Historically, hospital-based-task-oriented nursing programs post 1960s progressed to a professional model of nursing practice directed and grounded by legislation, regulations, standards of 
practice, code of ethics and nursing theory. High standard of patient care was afforded by these changes [12]. Team-based nursing was replaced with patient allocation models in which one nurse assumed responsibility for complete care of clients assigned [13]. The Nursing Task Force (1998) was established in response to growing concerns of nurses in their ability to provide safe care and nursing professional/governmental organizations about nursing workforce instability and predicted nursing shortage.

Team nursing reappeared in 2001-02 in NS' Nursing Strategy to address the perceived under-utilization of nurses in roles that maximize the use of their knowledge and skills MOHLC $[14,15]$. Of utmost importance to the proper and full-implementation of a well-structured model of nursing care is a clear understanding of the full scope of practice for each member on the healthcare team and the structuring of patient care as a team, not a mere division of labor and tasks [16]. Failure to adequately function as a team in all patient-care decisions reverts back to patient allocation. Now, however, LPNs are independently caring for patients within acute care settings, without the appropriate skillsets. In these circumstances it has been challenged that this new model of care can result in unsafe, incompetent, unethical care. It has been previously found that a greater ratio of RNs to LPNs on units results in lower hospital related deaths $[17,18]$.

Hence, cutting RN positions for the purpose of reducing the financial burden on the healthcare system may adversely affect patient health outcomes. Close examination of the trends in historical nursing reveals that this governmental "new model" of healthcare delivery is a reiteration of a previously failed model. The new model of care within Atlantic Canada threatens patient safety in its intention to remove the professional nurse from the bedside [12]. Provincial and national strategies to meet healthcare system needs involve optimizing conditions to work to full scope of practice as outlined in the 2002 Acts of the Colleges of RNs and LPNs of NS. In 2006, changes to the Acts, specifically addressing the definitions of nursing practice, were put forth to promote positive changes in scope of practice and scope of employment within a new model of care [15].

To review the issue that is presented is that nurses are required to work to full scope to promote client outcomes and job satisfaction. Yet, RN concerns over job security and increased responsibility in overseeing and being responsible for nursing care provided by LPNs at the bedside predominate [19]. The LPN scope of practice was delineated to impart cost-effectual, excellence in health care to the NS public. LPNs with the same number of year's experience of RNs receive approximately $\$ 10$ per hour less [20]. LPNs may be independently assigned clients with well-defined health challenges and predictable outcomes predictable outcomes. Clients with increased acuity or complexity of care and/or outcomes that are not predictable are dependently assigned to the LPN who works under the direction of a registered nurse to meet the care needs of the client [21].

Ineffectual team building and seamless integration of recommended policy guidelines into acute care best practices partly stem from deficient provider recognition of potential health and economic benefits associated with proper utilization [14]. An alternate explanation for nurses not working to their full scope of practice was identified in a Canadian study by Oelke et al. [7]. Barriers to working to full scope of practice included ineffective teamwork, poor communication among team members, and lack of time, heavy patient load and high patient acuity. Associated assumptions with an RN-led team-based approach to nursing are that the structural, financial and attitudinal barriers preventing RNs from practicing to their full scope have been removed [9].

\section{Alternate Leadership Strategies for Resolving the Issues}

Issues that nursing leaders are presented with include the challenges of being able to run a cost-efficient business, where in today's cutback on healthcare dollars LPNs are cheaper. The staffing decisions imparted by nurse managers prompted by patient health outcomes present a challenge in acute care settings due to high patient turnover. Nursing leaders must promote the value of the RN as an integral team member, such that staffing mixes must not compromise safe, efficient and ethical care as outlined as the primary value in the nursing code of ethics. In essence, knowledgeable leaders need to be visible and have a strong voice to inspire morale in support of professional nursing practice [22]. In today's changing fast-paced healthcare system to effectively confront change nurse leader competencies/skills must entail organizational management skills, relationship-building, effective communication skills, use of critical thinking skills and evidenced based practice to effect and promote change, creating effective relationships with other healthcare professionals and creating an atmosphere of shared leadership and shared decision making [23]. Strategies that a leader might endorse may be the contemporary 'staff meeting' style dealing with the here and now or the more dynamic transformational or visionary approach that will be applicable to future challenges. Both have equal but different pros and share the same financial barrier having funding in place to support change [24].

The issue that needs to be resolved is the need for role clarification and redesign of the work of health professionals. One leadership strategy could entail traditional supports including continuing education to maintain competencies. Besner identified continuing education of the current workforce as an important strategy in moving toward more effective healthcare teamwork in healthcare (2005). Facilitation of full scope practice involves team management, leadership support and support of continuing education to maintain competencies [7]. Under this strategy nurse managers would advocate for policies and have supports in place that permit RNs and LPNs to function to the maximum of professional practice abilities according to their respective provincial/territorial licensing body. To effectively incite change traditional scopes of practice must be reevaluated within the acute care setting to enable the delivery of services by the most qualified professional [3].

The Nursing environments: knowledge to action (NEKTA) study (2001-2005) was an initiative designed to measure the extent to 
which recommendations for positive change recommendations of national reports within Atlantic Canada in such areas as scope of practice were realized in the acute care setting [25]. NEKTA data indicate that the majority of respondents $(60 \%)$ surveyed from the Capital and Annapolis Valley Health Districts believe there has been no real change in the scope of practice of RNs, while $10 \%$ report the situation is worse and $23 \%$ report improvement. While recommendations appear in best practice and policy reports there has been little in the areas of knowledge dissemination and translation into practice despite seemingly effective leadership strategies.

A second leadership strategy might take a more dynamic or transformational approach [26,27], a strategic visionary model. Increased understanding of scope of practice may not be sufficient to incite effective team-building. One might actually have to take the perspective of the other to fully appreciate their role on the healthcare team. In this respect an effective leadership strategy must entail engaging discussion among team members about distinct and shared responsibilities to promote effective collaborative practice and enhanced quality of care [28]. In particular, in-service or training sessions would necessitate participation of both acute care RNs and LPNs in building collaborative working relationships to effectively respond to scope of practice issues in acute care settings [29]. The Beckwith Institute for Innovation in Patient Care outlines a transformational leadership strategy and evaluation methods (Appendix 2).

\section{Choice of Leadership Strategy and Justification}

The efficacy of a team-based approach in improving patient outcomes requires a systematic needs assessment particular to the healthcare setting with a clear understanding of roles of each of its members. Efficacy may be increased by focusing on the tasks at hand. Focus on the task at hand and achievement of nursing standards of performance with the day-to-day operations is characteristic of a transactional (mechanistic) leadership strategy Hibberd \& Smith [16]; Leatt \& Porter [30,31]. The model of team nursing was implemented and discarded in previous times. Transactional leadership strategies predominated. The theory behind the importance of the foundations has not changed which suggests that its success depends upon its delivery. Support from the public and policy makers as well as from individual teams members are required. Shared leadership increases the accountability of all team members for its success. Transformational leadership strategies that are interactive encompass all of the components of the traditional 'staff meeting' type of knowledge transfer but are more likely to be successful in the transformation of knowledge to practice. Theoretically, although nursing would be heavily involved it is also imperative that physicians be included.

Collectively nurses are well respected yet may require the endorsement of physicians [32] to gain the public's approval and purse. A visionary or transformational (organic) leadership strategy is necessary for successful implementation of working to full scope in nursing team practice including sharing their views with the public and policy developers [16,33]. Transformational leadership is similar to Oralando's model for nursing and a dynamic leader-follower relationship model. Directed by this strategy the leader bestows guidance for healthcare members to develop their own plan for patient care. Application of this model permits shared leadership in the planning of the organization and with it, responsibility for its success or failure ARNNL [10]. Strategic outcomes related to transformational leadership strategy result in financial savings, quality of care and flexibility in healthcare delivery [26,27]. Leadership competencies include public advocacy of reflective nursing practice [16]. Leaders must focus on meeting goals related to patient care. Evaluation criteria must include empirical evidence of lower mortality and morbidity rates associated with increased RN presence CNA, 2003 [34] (Appendix 3).

\section{Conclusion}

Leadership competencies and strategies are crucial to best promote team-based nursing and optimize patient outcomes. A visionary or transformational leadership strategy inspires teambuilding relationships and aspires to achieve maximal levels of performance within the scope of nursing practice.

\section{References}

1. Tonges MC (1989) Redesigning hospital nursing practice: The professionally advanced care team (ProAct) model. Journal of Nursing Administration 19(7): 31-38.

2. Weisman C (2010) Nursing practice models: research on patient outcomes.

3. (2002) Canadian Nursing Advisory Committee (CNAC) 2002. Our health, our future: Creating quality workplaces for Canadian nurses. Ottawa: Health Canada.

4. Mc Taggart ES (2007) Nurses' experiences of full scope LPN practice in acute care (T). University of British Columbia.

5. (2015) College of Registered Nurses of Nova Scotia (CRNNS) 2015. Interpreting and Modifying the Scope of Practice of the Registered Nurse.

6. (2014) College of Licensed Practical Nurses of Nova Scotia (CLPNNS) 2014. Guidelines for Licensed Practical Nurses in Nova Scotia.

7. Oelke ND, White D, Besner J, Doran D, McGillis Hall L, et al. (2008) Nursing workforce utilization: an examination of facilitators and barriers on scope of practice. Nursing Leadership 21(1): 58-71.

8. (2017) College of Registered Nurses of Nova Scotia (CRNNS) (2017). Effective Utilization of RNs and LPNs in a Collaborative Practice Environment.

9. O'Brien-Pallas L, Thomson D, Alksnis C, Bruce S (2001) The economic impact of nurse staffing decisions: Time to turn down another road? Hospital Quarterly 4(3): 42-50.

10. (2007) Association of Registered Nurses of Newfoundland and Labrador (ARNNL). Nursing leadership literature review.

11. Giddens J (2017) Transformational leadership: What every nursing dean should know. Journal of Professional Nursing. American Association of Colleges of Nurses.

12. Dewar K (2010) new model of nursing care a potential threat to patient safety. PEI. School of Nursing, Association of Nurses, Charlottetown, Canada, USA.

13. Fairbrother G, Jones A, Rivas K (2010) Changing model of nursing care from individual patient allocation to team nursing in the acute inpatient environment. Contemporary Nurse 35(2): 202-220. 
14. (2001) the Minister of Health and Long-Term Care (MOHL). Good nursing, good health; a good investment Progress report on the nursing task force strategy in Ontario.

15. (2006) Nova Scotia Health 2006. Nova Scotia's nursing strategy: A fiveyear summary report.

16. Hibberd J, Smith DL (2006) Nursing leadership and management in Canada. Toronto; Elsevier Canada.

17. Aiken LH, Clarke SP, Cheung RB, Douglas M Sloane, Jeffrey H Silber (2003) Educational levels of hospital nurses and surgical patient mortality. JAMA 290: 1617-1623.

18. Aiken LH, Sloane DM, Bruyneel L, Van den Heede K, Griffiths P, et al. (2014) RN4CAST consortium. Nurse staffing and education and hospital mortality in nine European countries: a retrospective observational study. Lancet 383(9931): 1824-1830.

19. McIntyre M, Thomlinson E, McDonald C (2005) Realities of Canadian nursing: Professional, practice, and power.

20. RN Canada (2009) Registered nurse salaries for nursing jobs in Canada.

21. (2005) College of Licensed Practical Nurses of Nova Scotia (CLPNNS) 2005 Scope of practice / Code of ethics / Standards of practice of licensed practical nurses of Nova Scotia.

22. (2003) Canadian Nurses Association (CNA). Position statement: staffing decisions for the delivery of safe nursing care.

23. Contino D (2004) Leadership competencies: knowledge, skills, and aptitudes nurses need to lead organizations effectively. Critical Care Nurse 24: 52-64.

24. Walker A, Olson R, Tytler S (2013) Collaborative nursing practice: RNs and LPNs working together. Canadian Nurse 109(6): 24-28.

This work is licensed under Creative Commons Attribution 4.0 License

Submission Link: https://biomedres.us/submit-manuscript.php
25. Leatt P, Porter J (2003) Where are the healthcare leaders? The need for investment in leadership development. Healthcare Papers 4(1): 14-31.

26. Wolf GA, Boland S, Aukerman M (1994a) A transformational model for the practice of professional nursing. Part 1, the model. The Journal of Nursing Administration 24(4): 51-77.

27. Wolf GA, Boland S, Aukerman M (1994b) A transformational model for the practice of professional nursing. Part 2, Implementation of the model. The Journal of Nursing Administration 24(5): 38-46.

28. Besner J, Doran D, McGillis Hall L, Giovannetti P, Girard F, et al. (2005) A systematic approach to maximizing nursing scopes of practice. Canadian Health Services Research Foundation.

29. Leiter MP (2006) Nursing Environments: Knowledge to action (NEKTA): Final report submitted to Health Canada, Health Policy Research Program: Quality workplaces for health professionals.

30. Porter-O'Grady T (2003a) A different age for leadership, part 1: New context: New content. Journal of Nursing Administration 33(2): 105110 .

31. Porter-O'Grady T (2003b) A different age for leadership, part 2: New rules new roles. Journal of Nursing Administration 33(3): 173-178.

32. Lewis S (2010) The last word. The Canadian Nurse.

33. (2010) Canadian Nurses Association (CNA) 2010. Ethics, relationships and quality practice environments.

34. (2003) Canadian Nurses Association (CNA). Position statement: staffing decisions for the delivery of safe nursing care.

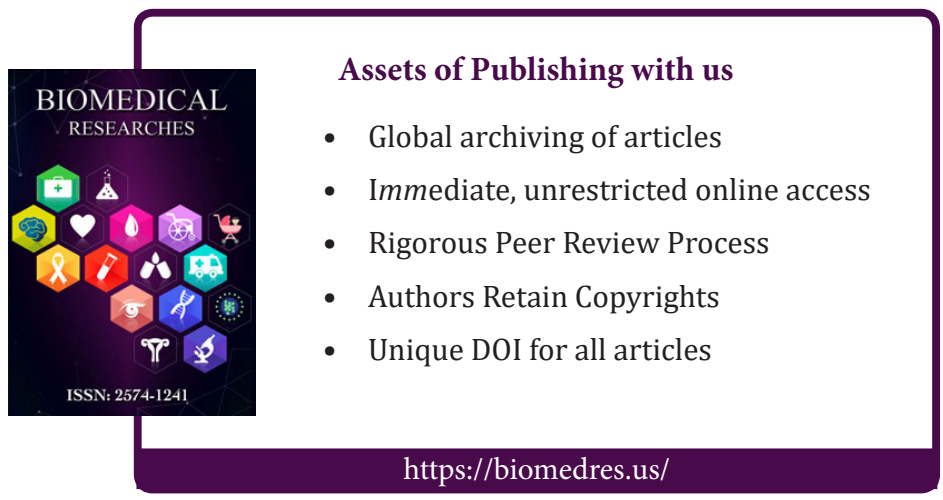

